\title{
A novel polyamidine-grafted
} carboxymethylcellulose: Synthesis, characterization and flocculation performance test

https://doi.org/10.1515/epoly-2019-0023

Received August 24, 2018; accepted November 13, 2018.

\begin{abstract}
This study facilitates the synthesis process of a novel graft copolymer (flocculant) using carboxymethyl cellulose, acrylonitrile and $\mathrm{N}$-vinyl formamide as raw materials. The carboxymethyl cellulose graft polyamidine (CMC-g-PAMD) can be used as new flocculant to replace the traditional polyacrylamide flocculant, which manifested its excellent flocculation and degradation efficiency. A five-membered cyclic copolymer was prepared by the graft copolymerization, and the synthesized flocculants were characterized by EA, TG-DTG, FT-IR, SEM and NMR, confirming the successful synthesis of the desired copolymers. The operation conditions for copolymerization were experimentally investigated, and the results indicated that the optimal initiator dosage, copolymerization temperature, amidinization temperature, acidification time and flocculant dosage were $4 \mathrm{~g} / \mathrm{L}, 50^{\circ} \mathrm{C}$, $90^{\circ} \mathrm{C}, 3 \mathrm{~h}$ and $60 \mathrm{mg} / \mathrm{L}$, respectively. Compared with the traditional polyacrylamide flocculant, the CMC-g-PAMD presented an outstanding flocculation ability of $96.1 \%$ under its optimal operation conditions, which showed an enormous potential in the application of coalmine wastewater treatment.
\end{abstract}

Keywords: graft copolymer; copolymerization; flocculant; amidinization; wastewater treatment

* Corresponding author: Yuqi Wang, School of chemical Engineering, Northwest University, Xi'an, Shaanxi, China; Research Institute of Shaanxi Yanchang Petroleum, Xi'an, Shaanxi, China e-mail: wangyuqi@nwu.edu.cn.

Yijun Yue, Lan Zheng, Shanshan Li, Xiaolong Han and Le Wu, School of chemical Engineering, Northwest University, Xi'an, Shaanxi, China; Research Institute of Shaanxi Yanchang Petroleum, Xi'an, Shaanxi, China.

Jinqiao Wu, Research Institute of Shaanxi Yanchang Petroleum, Xi'an, Shaanxi, China.

\section{Introduction}

Nowadays the world's economic and social development have a great dependence on fossil energy, which is still increasing with rapid scientific and technological progress $(1,2)$. Coal is one of the fundamental fossil energy sources in the world. In 2016, China's coal production and consumption were 2.41 billion tons and 2.70 billion tons, respectively (3). Although coal as a traditional energy has favored and improved human life greatly, it will inevitably produce large amounts of sewage water during the mining \& utilization process $(4,5)$. If the mentioned wastewater were emitted directly without any treatment, the natural environment would suffer serious or even irreparable damage $(6,7)$. The most convenient and effective method is to use flocculant for wastewater treatment (8), and the conventional flocculants are divided into two major categories: inorganic (9) and organic flocculants (10). Inorganic flocculants (11) include iron salt (12), aluminum salt (13), polyaluminum chloride (PAC) (14) and Polysilicate (15). Although inorganic flocculants are usually cheap and economic, they have several disadvantages such as large dosage, low flocculation efficiency and strong corrosion. Organic flocculants are divided into natural polymers and artificially synthesized polymers. Meanwhile the artificially synthesized polymers mainly include polyacrylate (16), polydiallyldimethylammonium chloride (PDADMAC) (17) and polyacrylamide (PAM) (18), which behave a high turbidity removal effect, small dosage and weak corrosion. However, most synthesized polymers using as flocculants are poisonous and harmful, which are not environmental-friendly and difficult to be degraded naturally. Faced with these situations, many copolymers can be produced by grafting artificial polymers $(19,20)$ onto the hydroxyl groups of natural polymers $(21,22)$. Zhou et al. (23) synthesized a biocopolymer by starch grafting with acrylamide (AM) and dimethyl diallyl ammonium chloride (DMDAAC) through 
an initiator system of $\mathrm{KMnO}_{4} / \mathrm{HIO}_{4}$. The results showed the graft starch can remove reactive dyes and disperse dyes from wastewater effectively, and the dye removal efficiency of the grafted starch was nearly 10\% higher than PAM. Huang et al. (24) prepared the quaternary ammonium salt grafted starch flocculants, starch-graftpoly(2-methacryloyloxyethyl) trimethyl ammonium chloride (St-g-PDMC), and the efficiencies St-g-PDMCs for flocculation of kaolin and Escherichia coli suspensions as well as their mixtures were systematically examined in laboratory scale. Experimental results indicated that St-gPDMCs exhibited dual functionality of high flocculation effects and antibacterial properties.

In our previous work, Zou et al. (19) proposed a cationic pea starch grafted acrylamide as a flocculant, and investigated its application in coalmine wastewater treatment. The results showed that the graft polymer has several superior application properties compared with PAM flocculant; Li et al. (20) developed a few of graft copolymers of different legume starches and acrylamide, and the experimental results indicated the graft copolymer of acrylamide and mung bean starch behaved an outstanding flocculation effect than other flocculants. It was revealed that legume starch with higher amylose content can achieve better flocculation performance. Compared with traditional PAM flocculant, these St-g-AM copolymers improved both flocculation and biodegradation ability significantly. However, starch is one of the important foods for mankind and animals, and when starch is used as a raw material to produce St-g-AM flocculant, it would inevitably reduce the global food supply and restrict its further application. Additionally, during the copolymerization of St-g-AM, small amount of AM could reside inside the flocculant which is toxic and harmful to people's health. Since cellulose is characterized by its wide material sources which has similar structure and property with starch, it can be considered to replace starch and prepare novel cellulose grafted copolymer using as flocculant.

Polyamidine (PAMD) is an organic compound containing amidine groups in polymer molecular chain, which possesses high charge density and has been used in many fields (25), such as the capture of $\mathrm{CO}_{2}(26)$ and photosensitive material (27). According to the current literature (28), PAMD performed a better treatment effect than PAM in water treatment field, avoiding the toxicity and pollution of acrylamide monomer as an ideal flocculant. Therefore, using carboxymethylcellulose (CMC) \& PAMD for copolymerization can solve the aforementioned difficulties and produce a high efficiency and environmental-friendly flocculant.
In this work, the CMC-g-PAMD was prepared and investigated, and the related work has applied for China invention patent (29). The copolymer was characterized by elemental analysis (EA), thermogravimetric analysis (TG-DTG), Fourier transform infrared (FT-IR), scanning electron microscopy (SEM) and nuclear magnetic resonance (NMR). Moreover, the flocculation performance was experimentally evaluated and compared by coalmine wastewater treatment.

\section{Experimental}

\subsection{Materials and instruments}

The following reagents were used in this experiment: carboxymethyl cellulose (CMC;Henan Weidao Chemical Products Co., Ltd.), ceric ammonium nitrate (CAN; Sinopharm Chemical Reagent Co., Ltd.), N-vinyl formamide (NVF; Sigma-Aldrich Co., Ltd.), acrylonitrile (AN; Sinopharm Chemical Reagent Co., Ltd.), 2,2'-Azobis (2-methylpropionamidine) dihydrochloride (AIBA, Aladdin Reagents Co., Ltd.), hydrochloric acid (Sichuan Xilong Chemical Co., Ltd.), anhydrous ethanol (Tianjin Fuyu Fine Chemical Co., Ltd.), polyacrylamide (PAM; Sinopharm Chemical Reagent Co., Ltd.), sodium hydroxide (Tianjin Hongyan Reagent Factory). The instruments used for the sample analysis were listed as follows: EA (Vario Macro Cube, Germany Element, Inc.), TGA-DTG (Discovery, U.S. TA Instruments), FT-IR spectrometer (Vertex 7.0, Germany Brukern Optics), SEM (EVO MA10, Germany Zeiss, Inc.), NMR (liquid-phase AV $400 \mathrm{MHz}$ and solidphase AV $600 \mathrm{MHz}$, Germany Bruker Optics, Inc.). The ${ }^{1} \mathrm{H}$-NMR spectrum was recorded with dimethyl sulfoxide (DMSO) as the solvent, and ${ }^{1} \mathrm{H}$ MAS and ${ }^{13} \mathrm{C} \mathrm{CP} / \mathrm{MAS}$ NMR were experimentally performed using a $4 \mathrm{~mm}$ MAS probe and a spinning rate of $12 \mathrm{kHz}$ at a resonance frequency of $600.1 \mathrm{MHz}$ and $150.9 \mathrm{MHz}$, respectively. TGA-DTG was constructed within $50-600^{\circ} \mathrm{C}$ at a heating rate of $10^{\circ} \mathrm{C} /$ min under a certain constant $\mathrm{N}_{2}$ gas flow, and both mass of the substance and the thermal decomposition were determined as a function of temperature.

The procedure and mechanism of CMC-g-PAMD synthesis were described in Figure 1. Copolymerization and amidinization can proceed smoothly in water under a $\mathrm{CO}_{2}$ atmosphere. $1 \mathrm{~g} \mathrm{CMC}$ and $50 \mathrm{~mL}$ deionized water were added into a 250 -mL three-neck flask equipped with blender, condenser and $\mathrm{CO}_{2}$ inlet pipe, and the mixture was stirred under a thermostat water bath for $1 \mathrm{~h}$ at $40-60^{\circ} \mathrm{C}$. Then the NVF, AN and initiator (CAN:AIBA $=1: 1$ ) 
A
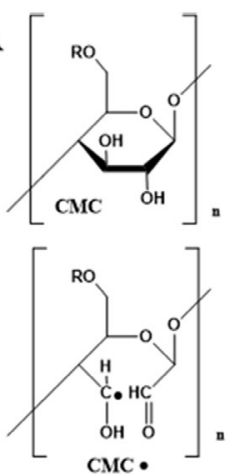

CMC .

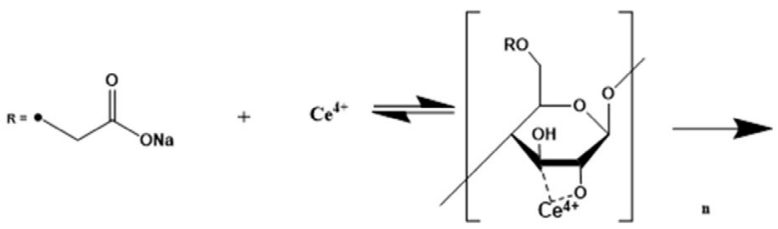

OR

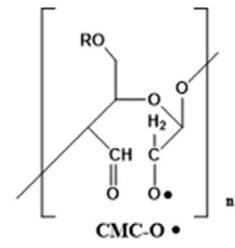

$+\mathrm{Ce}^{3+}+\mathrm{H}^{+}$

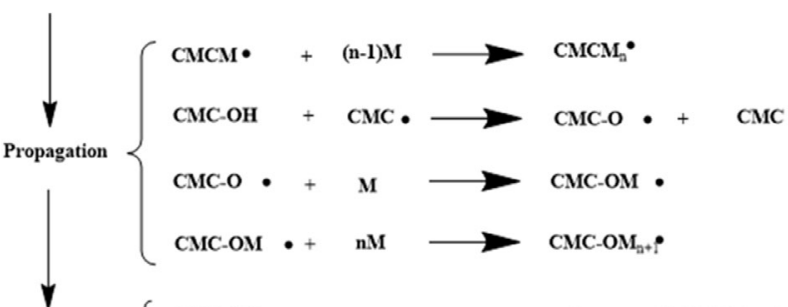

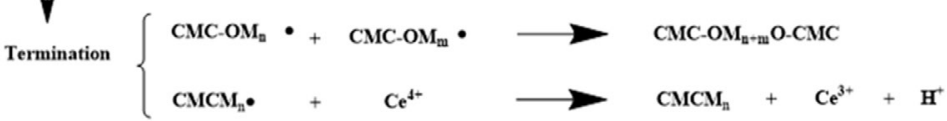

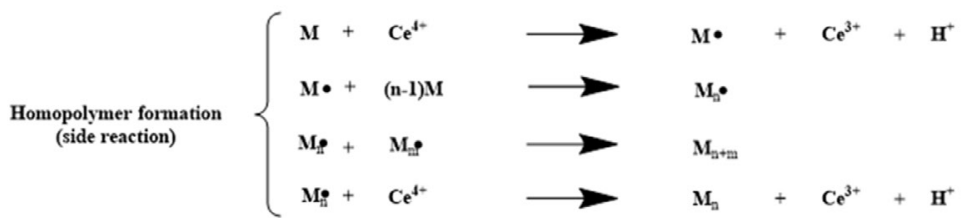

B
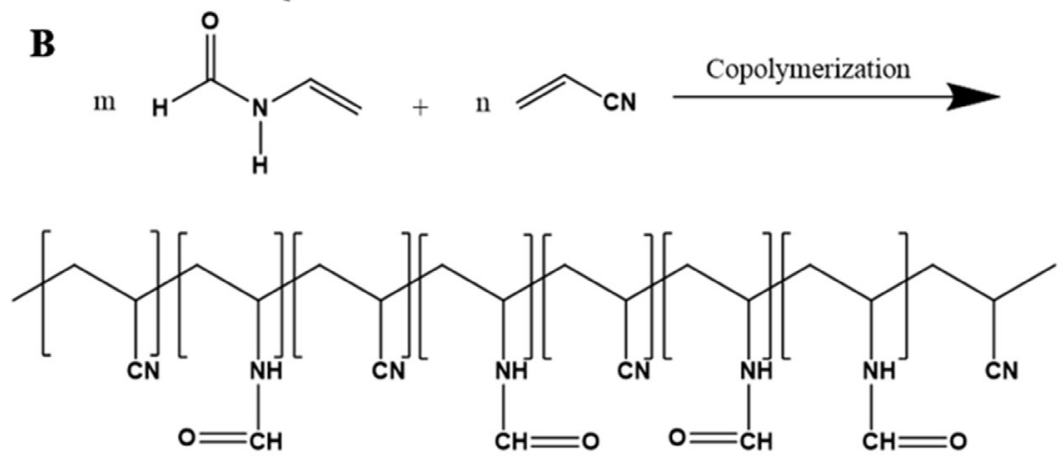

Hydrolysis under acidic conditions

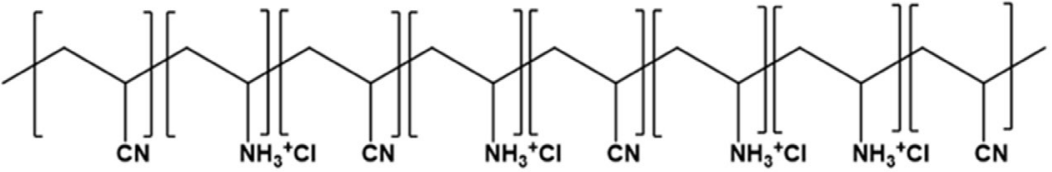

Amidinze

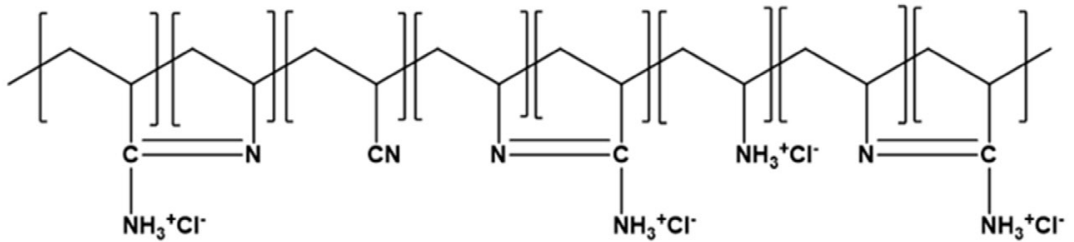

Figure 1: The procedure and mechanism of grafting copolymerization (a); amidinization (b). 
were injected into the flask and kept stirring at $40-60^{\circ} \mathrm{C}$ for $5 \mathrm{~h}$. Afterwards the concentrated hydrochloric acid was dropped in the reactants at desired times during the copolymerization, and kept theamidinization of copolymers at $80-100^{\circ} \mathrm{C}$ for $3 \mathrm{~h}$. Later the copolymer samples were extracted using anhydrous ethanol, and the precipitated crude products were obtained after vacuum drying.

Finally the new flocculant (CMC-g-PAMD) samples could be obtained after removing the impurities inside the crude products by the following process: firstly the crude products were washed with acetone; secondly the samples was smashed into powder after drying under vacuum; finally the "Varma method" was used to reflux the crude samples in a Soxhlet extractor for $12 \mathrm{~h}$, using a mixed solvent of glacial acetic acid and ethylene glycol $(1: 1 \mathrm{v} / \mathrm{v})$, which could remove the unreacted PAMD and monomers from the crude products effectively.

\subsection{Evaluation of the grafting efficiency (GE)}

GE was calculated according to the following equation:

$$
G E=\frac{M_{p}-M_{c}}{M_{c}} \times 100 \%
$$

where $M_{c}$ denotes the mass of CMC (g), $M_{p}$ represents the mass of the product purified by Soxhlet extractor (g).

\subsection{Flocculation performance evaluation}

A certain amount of the flocculant and $50 \mathrm{ml}$ of coalmine wastewater were put into a $50 \mathrm{ml}$ beaker and stirred for $1 \mathrm{~min}$, and the transmittance of supernatant was measured with spectrophotometer at the wavelength of $610 \mathrm{~nm}$. The coalmine wastewater sample was taken from Shanxi Ximing Coal Group Co., Ltd., and the detailed information was given below: $\mathrm{pH}=7.90$, turbid degree $=4800 \mathrm{NTU}, \mathrm{COD}$ (chemical oxygen demand) $=89 \mathrm{mg} / \mathrm{L}$, SS (suspended substance) $=$ $1120 \mathrm{mg} / \mathrm{L}$, iron ion $=8.93 \mathrm{mg} / \mathrm{L}$, manganese ion $=7.75 \mathrm{mg} / \mathrm{L}$.

\section{Characterizations of the} CMC-g-PAMD

\subsection{EA}

The EA results of AN, NVF, CMC, and the new flocculant (CMC-g-PAMD) were given in Table 1. It was apparent from the table that the $\mathrm{C}, \mathrm{H}, \mathrm{O}$ and $\mathrm{N}$ contents of the
Table 1: Elemental analysis.

\begin{tabular}{lllrr}
\hline Item & C (\%) & H (\%) & N (\%) & O (\%) \\
\hline AN & 67.77 & 4.910 & 27.53 & 0 \\
NVF & 47.42 & 6.286 & 18.60 & 27.69 \\
CMC & 36.40 & 5.638 & 0 & 57.95 \\
Prepared sample & 56.47 & 5.466 & 17.51 & 20.55 \\
\hline
\end{tabular}

new flocculant were among those of AN, NVF, CMC and the prepared sample. Compared with CMC, a significant increase of nitrogen percentage inside the prepared sample can be observed, suggesting the successful grafting of AN and NVF onto the backbone of CMC. It can be concluded from Table 1 that PAMD was actually grafted onto CMC during the copolymerization, and produced the new flocculant with specific features and performances.

\subsection{TG-DTG}

The TGA-DTG curves of CMC and the new flocculant (CMCg-PAMD) under a nitrogen atmosphere were shown in Figure 2. From the curves we can clearly see the loss of humidity, phase transition and degradation processes. For CMC, three weight loss stages were observed: the first stage of weight loss from room temperature to $180^{\circ} \mathrm{C}$ was supposed to be derived from water loss; the next weight loss stage within $200-400^{\circ} \mathrm{C}$ was due to broken of hydroxyl group; and the last weight loss stage at 400$530^{\circ} \mathrm{C}$ was attributed to the rupture of the main chains of CMC and key bonds. In the case of CMC-g-PAMD, the weight loss during the first region $\left(46-190^{\circ} \mathrm{C}\right)$ was related to moisture removal; a sudden large weight loss occurred within $200-360^{\circ} \mathrm{C}$ as the temperature rose, indicating the thermal decomposition of CMC; and the final weight loss stage $\left(360-520^{\circ} \mathrm{C}\right)$ could be explained as the degradation of PAMD chains. As shown in Figure 2, the new flocculant had more residue (37\%) than that of CMC (29\%) after high temperature heating, and performed a better thermal stability, which illustrated the successful grafting of PAMD onto CMC.

\subsection{FT-IR}

FT-IR was used to characterize the functional groups of product (CMC-g-PAMD), and the results were shown in Figure $2 \mathrm{~b}$. From the figure we can witness that the broad peak of CMC occurred at $3311 \mathrm{~cm}^{-1}$, which was assigned to the stretching vibrations of $\mathrm{O}-\mathrm{H}$ (30). The peak at $1017 \mathrm{~cm}^{-1}$ represented the functional groups of $\square$. For the NVF spectrum in Figure $2 b$, we can observe that the 

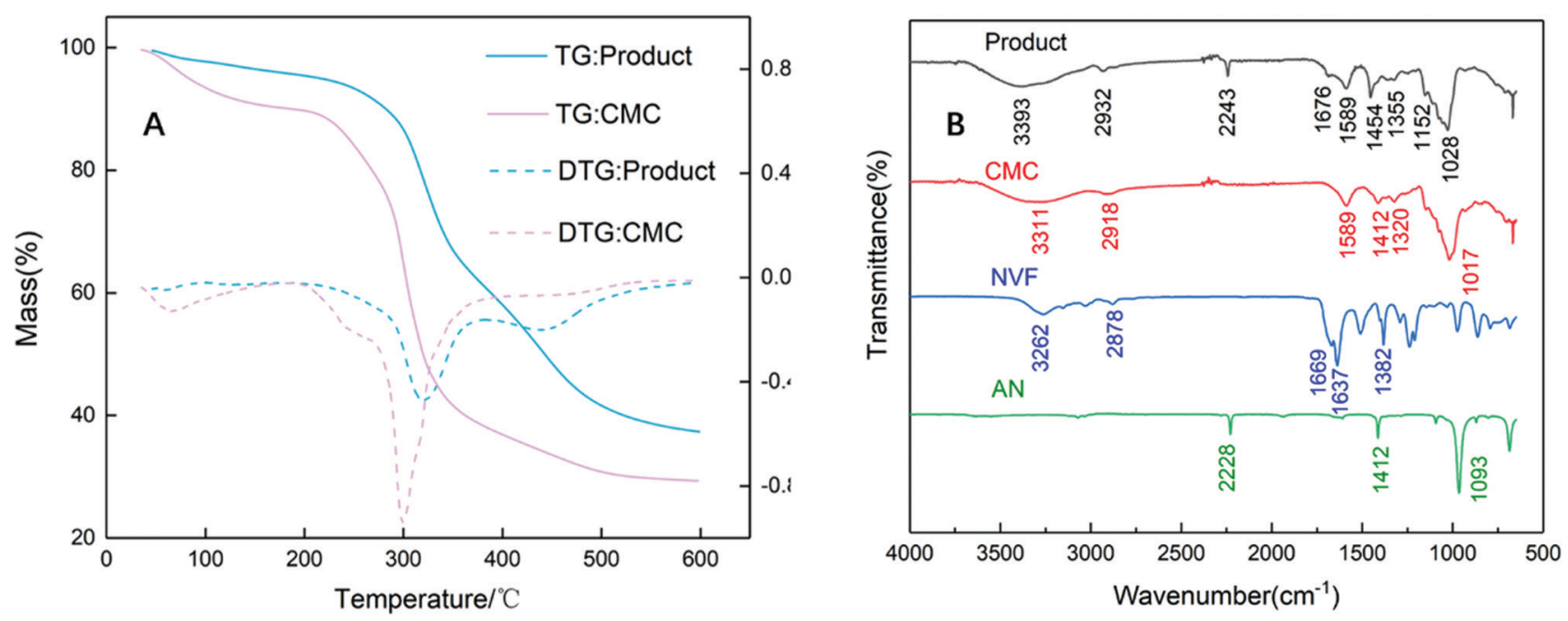

Figure 2: (a) TG-DTG curves of the prepared sample; (b) FT-IR of CMC, NVF, AN and the product.

bands at 3262 and $1382 \mathrm{~cm}^{-1}$ were corresponding to $\mathrm{N}-\mathrm{H}$ and $\mathrm{C}-\mathrm{N}$ stretching vibration peaks in amide group respectively, and a stretching vibration peak of $\mathrm{C}=\mathrm{O}$ appeared at $1669 \mathrm{~cm}^{-1}$ (31). For the AN spectrum in Figure $2 \mathrm{~b}$, the peak at $2228 \mathrm{~cm}^{-1}$ (AN) confirmed the existence of $\mathrm{C} \equiv \mathrm{N}$ (32). Obviously, compared with CMC, NVF and AN, there are some differences in the infrared spectrum of the product. From the infrared spectrum of the product, the $\mathrm{O}-\mathrm{H}$ stretching band of $\mathrm{CMC}$ backbone and the $\mathrm{N}-\mathrm{H}$ stretching band of the NVF chain overlapped each other, leading to a combined peak of $\mathrm{O}-\mathrm{H}$ and $\mathrm{N}-\mathrm{H}$ groups for the prepared flocculant at $3393 \mathrm{~cm}^{-1}$. Meanwhile, the peak of product at $2243 \mathrm{~cm}^{-1}$ was mainly due to the characteristic absorption of $\mathrm{C} \equiv \mathrm{N}$, confirming the participation of $\mathrm{AN}$ in the graft copolymerization. Compared with peak at $1669 \mathrm{~cm}^{-1}$ (NVF), the peak at $1676 \mathrm{~cm}^{-1}$ of the new flocculant was ascribed to the $\mathrm{C}=0$ stretching vibrations (29), and the peak area decrease could also verify the hydrolysis of $\mathrm{C}=\mathrm{O}$ in NVF. Moreover, the stretching vibrations of $\mathrm{C}-\mathrm{N}$ and $\mathrm{C}=\mathrm{N}$ were observed at $1454 \mathrm{~cm}^{-1}$ and $1152 \mathrm{~cm}^{-1}$, respectively, demonstrating the occurrence and development of amidinization reaction as expected (33).

\subsection{SEM}

Figure 3 showed the SEM micrographs of the new flocculant (Figures 3a and 3b) and CMC (Figures 3c and 3d). It was obvious that the morphological characteristics of the product varied significantly after the reaction (Figure 3b vs. Figure 3d), and the original crystal form of CMC (Figure 3b vs. Figure 3d) turned into the amorphous state of the product (Figures $3 \mathrm{a}$ and $3 \mathrm{~b}$ ). It can be revealed from the Figure $3 \mathrm{~b}$ that the new flocculant was uniform fine powder and these small granules showed a net-like structure under greater magnification. Since the smaller particles of flocculant samples tended to present higher diffusion rate in aqueous solution, the net structure of the product may promote the net capture effect remarkably, resulting in an outstanding flocculation performance accordingly.

\subsection{NMR}

The ${ }^{13} \mathrm{C}$ NMR and ${ }^{1} \mathrm{H}$ NMR spectra of the new flocculant were experimentally conducted, and the results were shown in Figure 4. It can be revealed from the figure that the highest chemical shift ( $\delta$ 3.7) in the ${ }^{1} \mathrm{H}$ NMR spectrum (Figure 4a) could be assigned to $-\mathrm{NH}_{2}$ group. The peaks at chemical shifts ( $\delta 3.2$ ) can be ascribed to $\mathrm{H} 2, \mathrm{H} 5$ protons on the CMC backbone, and the chemical shift ( $\delta 3.4$ ) could be described as the protons of $\mathrm{H} 7$ on the carboxymethyl. The peak at chemical shifts of $\delta 2.1$ can be corresponding to $\mathrm{H} 1, \mathrm{H} 3$ and H4; $\delta 1.3, \delta 1.4, \delta 2.0$ and $\delta 2.5$ ascribed to H1O, H11, H6 and H9 respectively. For the ${ }^{13} \mathrm{C}$ NMR spectrum (Figure 4b), the peaks at $\delta 108.6$ and $\delta 76.0$ were assigned to $\mathrm{C} 1$ and $\mathrm{C} 10$ respectively, and the peak at $\delta 121.6$ ascribed to $\mathrm{C} 3$ and C4. Meanwhile the peak at $\delta 201.4$ attributed to $\mathrm{C} 8$ and $\mathrm{C} 13$, and the peak at $\delta 44.1$ ascribed to $\mathrm{C} 2, \mathrm{C} 5, \mathrm{C} 7$, C9 and C12.

\section{Optimization of reaction conditions}

\subsection{Effect of initiator dosage}

Since initiator is crucial for graft copolymerization, the proper type and dosage of initiator can significantly 

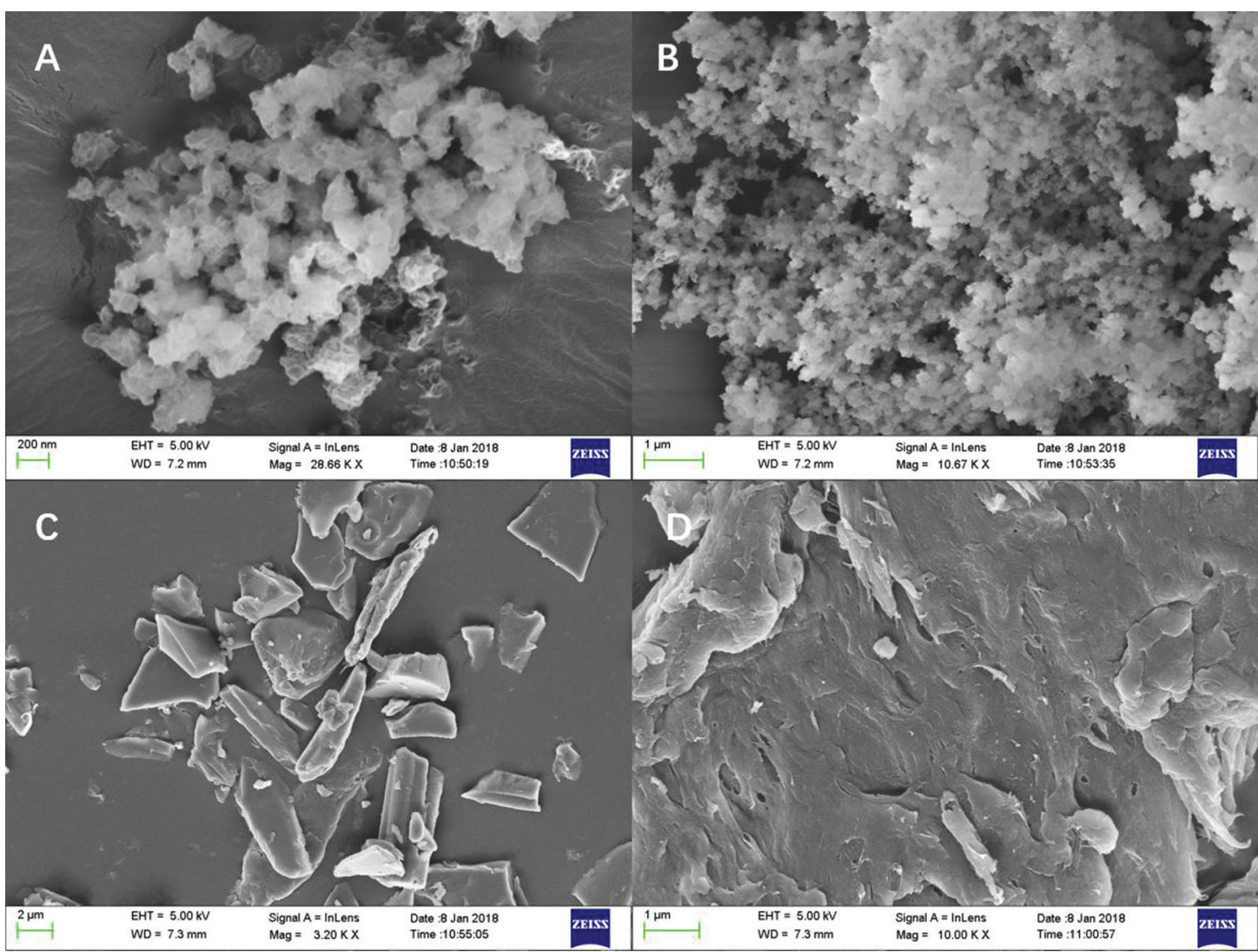

Figure 3: SEM photographs of (a) CMC-g-PAMD ( $\times 50000)$; (b) CMC-g-PAMD $(\times 10000)$; (c) CMC $(\times 5000)$; (d) CMC $(\times 10000)$.
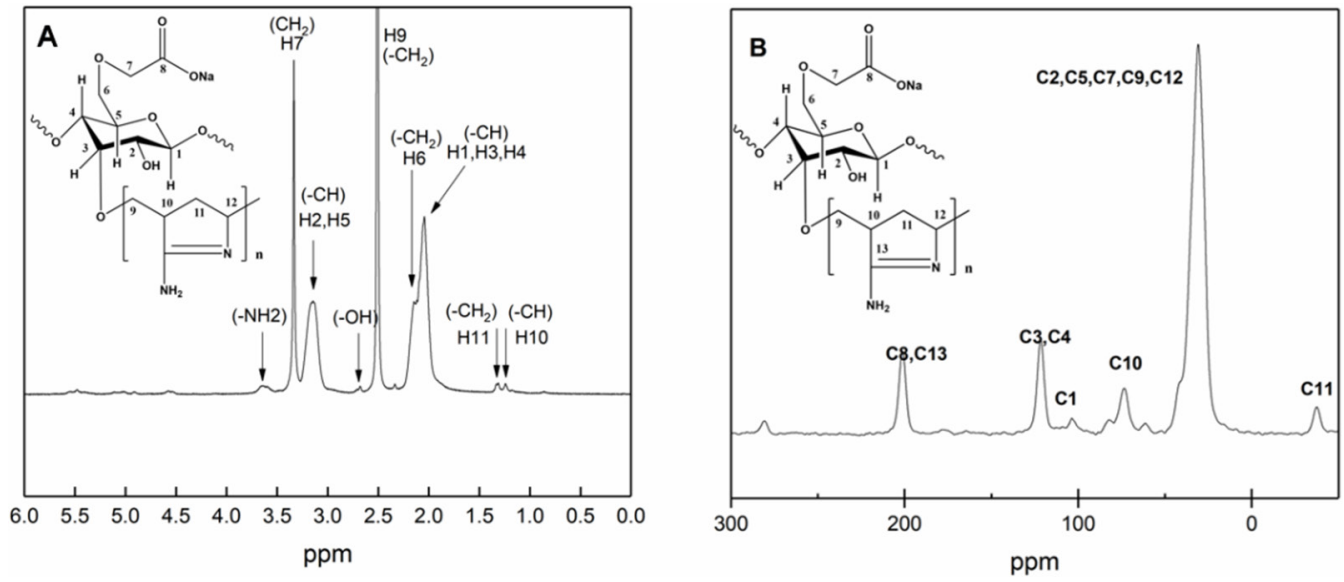

Figure 4: (a) ${ }^{1} \mathrm{H}-\mathrm{NMR}$ of the new flocculant; (b) ${ }^{13} \mathrm{C}-\mathrm{NMR}$ of the new flocculant.

promote the reaction and should be investigated intensively. Zhao et al. (34) studied different initiators for the copolymerization of NVF, AN and starch, and the optimal initiator for copolymer synthesis was determined as a mixture of CAN + AIBN (m:m = 1:1). Hence we used the initiator in this work, and further investigated its optimal addition amount for the graft copolymerization of the new flocculant. Different initiator dosage from
$1 \mathrm{~g} / \mathrm{L}$ to $5 \mathrm{~g} / \mathrm{L}$ was experimentally studied, and results were shown in Figure 5a. It can be seen from the figure that the transmittance of wastewater samples climbed gradually with increasing initiator dosage at initial stage, and the flocculation effect reached its peak at the optimal initiator dosage of $4 \mathrm{~g} / \mathrm{L}$; afterwards the transmittance tended to drop. This is because the radical polymerization can proceed efficiently when the initiator concentration 

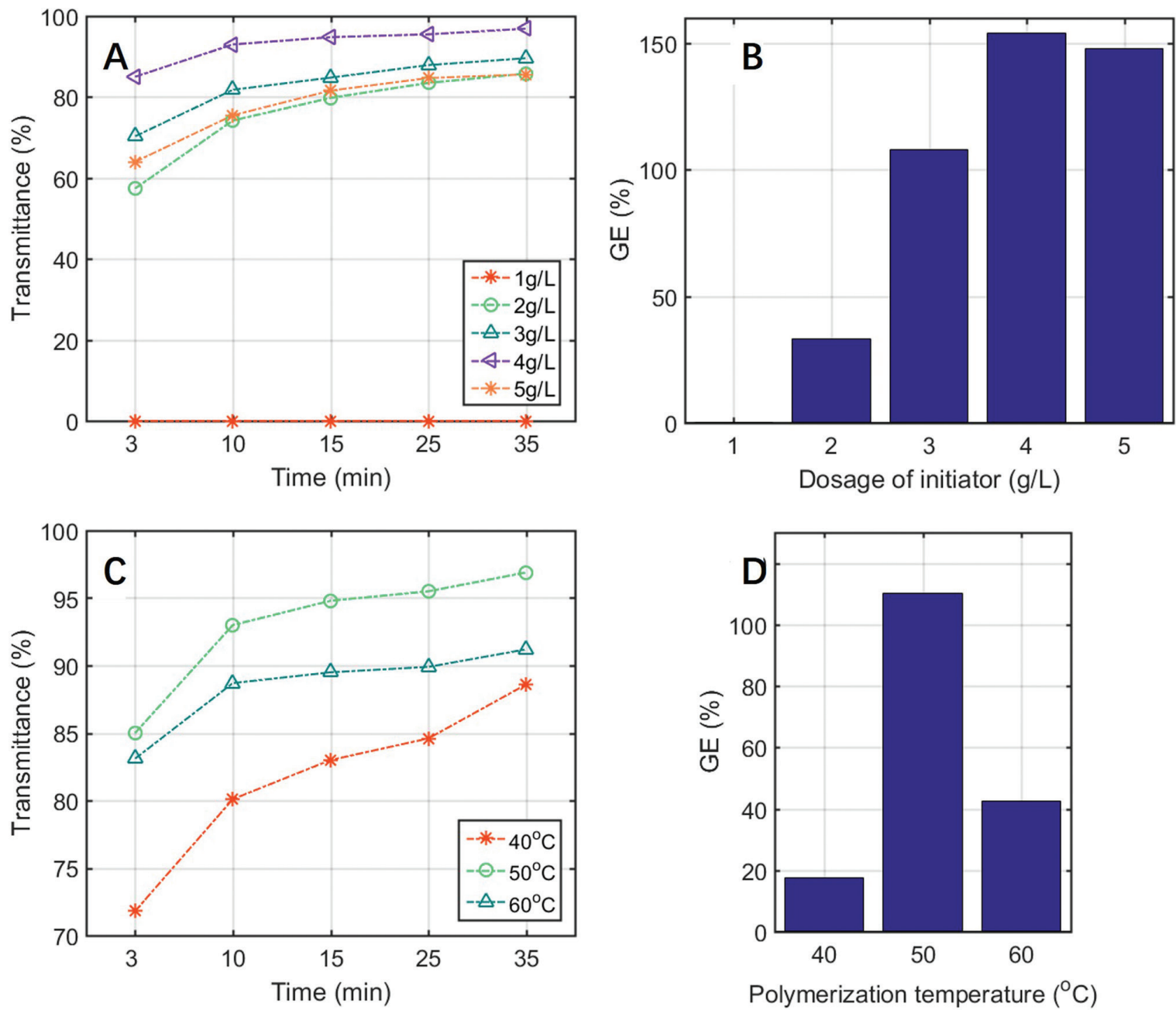

Polymerization temperature $\left({ }^{\circ} \mathrm{C}\right)$
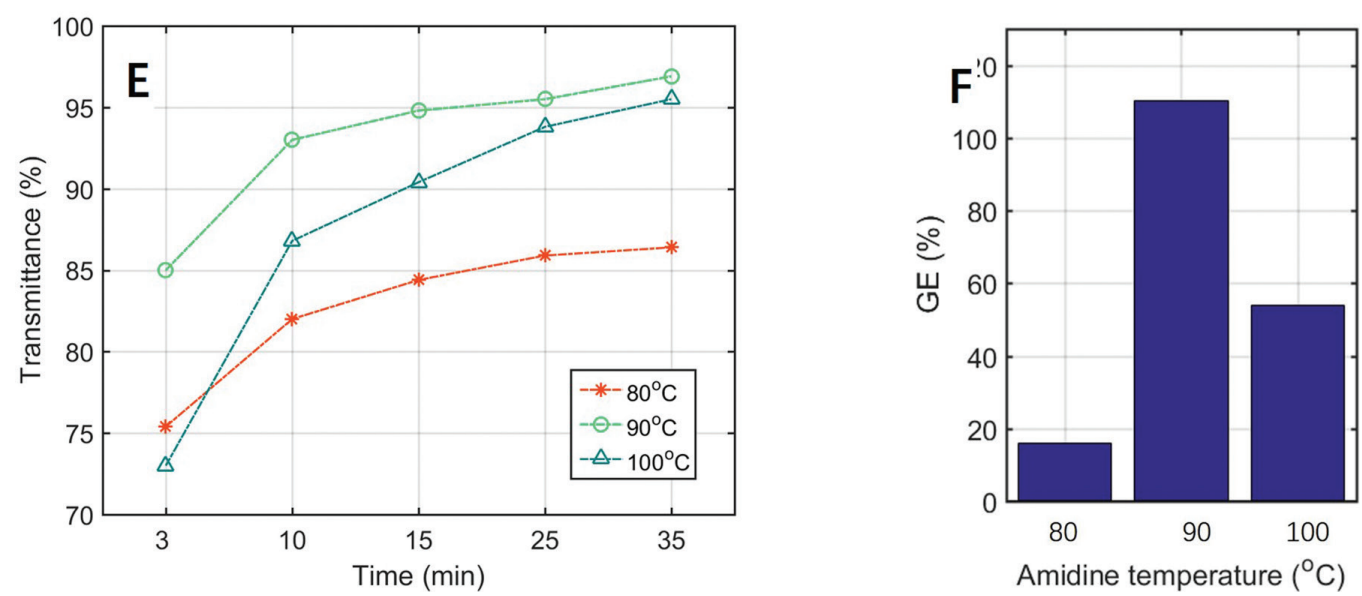

Figure 5: Effect of initiator dosage on transmittance (a) and GE (b); effect of polymerization temperature on transmittance (c) and GE (d); effect of amidinization temperature on transmittance (e) and GE (f).

is sufficiently high, and the initiator concentration of $4 \mathrm{~g} / \mathrm{L}$ was proved to have the most excellent flocculation performance. Moreover, the double bond $(\mathrm{C}=\mathrm{C})$ activity of AN was obviously higher than that of NVF in the free radical polymerization due to its relatively small steric hindrance, so the excessive initiator concentration could enhance the homopolymerization of AN and hinder the copolymerization between NVF and AN, resulting in the reduced amidine content and poor flocculation effect. Similarly, as shown in Figure 5b, the product gained the 
largest GE of $156 \%$ under the initiator dosage of $4 \mathrm{~g} / \mathrm{L}$, meanwhile the over high initiator addition would impel the premature termination of polymerization chains, causing the GE decrease of the CMC-g-PAMD.

\subsection{Effect of copolymerization temperature}

In Figures $5 c$ and $5 d$, the influence of copolymerization temperature was presented. From the figure we can witness that both GE and transmittance showed the same trend: increase first and then decrease. Before $50^{\circ} \mathrm{C}$, the reaction rate ascended steadily as graft copolymerization temperature extended. When the temperature exceeded $50^{\circ} \mathrm{C}$, the side reactions including homopolymerization was accelerated remarkably, which can greatly affect the copolymerization and flocculation ability, leading to the evident decline of GE and transmittance accordingly. Thus, $50^{\circ} \mathrm{C}$ was determined as the optimal copolymerization temperature.

\subsection{Effect of amidinization temperature}

The amidinization temperature has great impact on the molecular diffusion, GE and flocculation performance, and a few experiments were carried out to explore the optimal temperature. As shown in Figures 5e and 5f, when the amidine temperature was below $90^{\circ} \mathrm{C}$, the $\mathrm{GE}$ and flocculation performance of the CMC-g-PAMD ascended greatly as temperature extended. This was largely due to the increase of both molecular diffusion and amide hydrolysis (to produce amino group) rates, which could promote the driving force for the copolymerization. Whereas, both GE and flocculation efficiency tended to decline when theamidinization temperature exceeds $90^{\circ} \mathrm{C}$. This could be because an excessively high temperature might cause a cross-linking reaction of the amino group generated by hydrolysis, consuming the amino group as the raw material for amidinization reaction, and thus the amidine content of the product could be reduced and the flocculation effect was deteriorated evidently. Additionally, high amidinization temperature may also cause the chain breaking of the copolymerized products, resulting in an obvious decrease of GE. Hence $90^{\circ} \mathrm{C}$ was selected as the optimal amidinization temperature.

\subsection{Effect of acidification time}

The acidification time is defined as the actual copolymerization time after the addition of hydrochloric acid. As shown in Figures $6 \mathrm{a}$ and $6 \mathrm{~b}$, we can clearly observe the optimal acidification time was confirmed to be $3 \mathrm{~h}$, which demonstrated an overwhelming flocculation performance than any other acidification time. More or less acid treat time was unfavorable to the transmittance and GE, and both copolymerization efficiency and flocculation ability dropped significantly when the acid treat time exceeded $3 \mathrm{~h}$. The reason was because NVF has extremely poor water solubility, and the ammonium salt was formed and the NVF solubility can be improved significantly after the treatment of hydrochloric acid, causing the stimulating effect on the copolymerization and flocculation. However, the polysaccharide in the product tended to hydrolyze apparently when the acidification time exceeded $3 \mathrm{~h}$, leading to the decrease of GE and flocculation performance of the prepared samples.

\subsection{Effect of flocculant dosage}

Figure 6c showed the impact of dosage of the synthesized flocculant on its transmittance under the optimal operation conditions. As show in Figure 6c, the flocculation performance increased steadily with the rise of the flocculant dosage at initial stage, afterwards the transmittance tended to decline obviously, and the experimental results indicated that the transmittance was over $95 \%$ under the optimal flocculant dosage of $60 \mathrm{mg} / \mathrm{L}$. This could be explained as follows: since the new flocculant has dual effects on neutralization and flocculation, it is more prone to collect the suspended particles inside coalmine wastewater with increasing adding amount (35). Meanwhile the flocculation effect tended to decline when the flocculant dosage exceeded its optimal amount, which was not only because the over-neutralized electric charges of wastewater can make the like charges repelled each other, but also the impurity particle surface would be covered by superfluous copolymer chains, leading to the obstacle for bridging flocculation (36).

Furthermore, the flocculation efficiency between the product and conventional PAM were experimentally compared, and the results were shown in Figure 6d. It can be concluded from the figure that the new flocculant behaved a dominate treatment effect for coalmine waste water than PAM under its optimal operation conditions, which would have a great potential and promising application for waste water treatment in the future.

\section{Conclusion}

In this work, a new flocculant (CMC-g-PAMD) was produced by the graft copolymerization of $\mathrm{AN}, \mathrm{NVF}$ and 

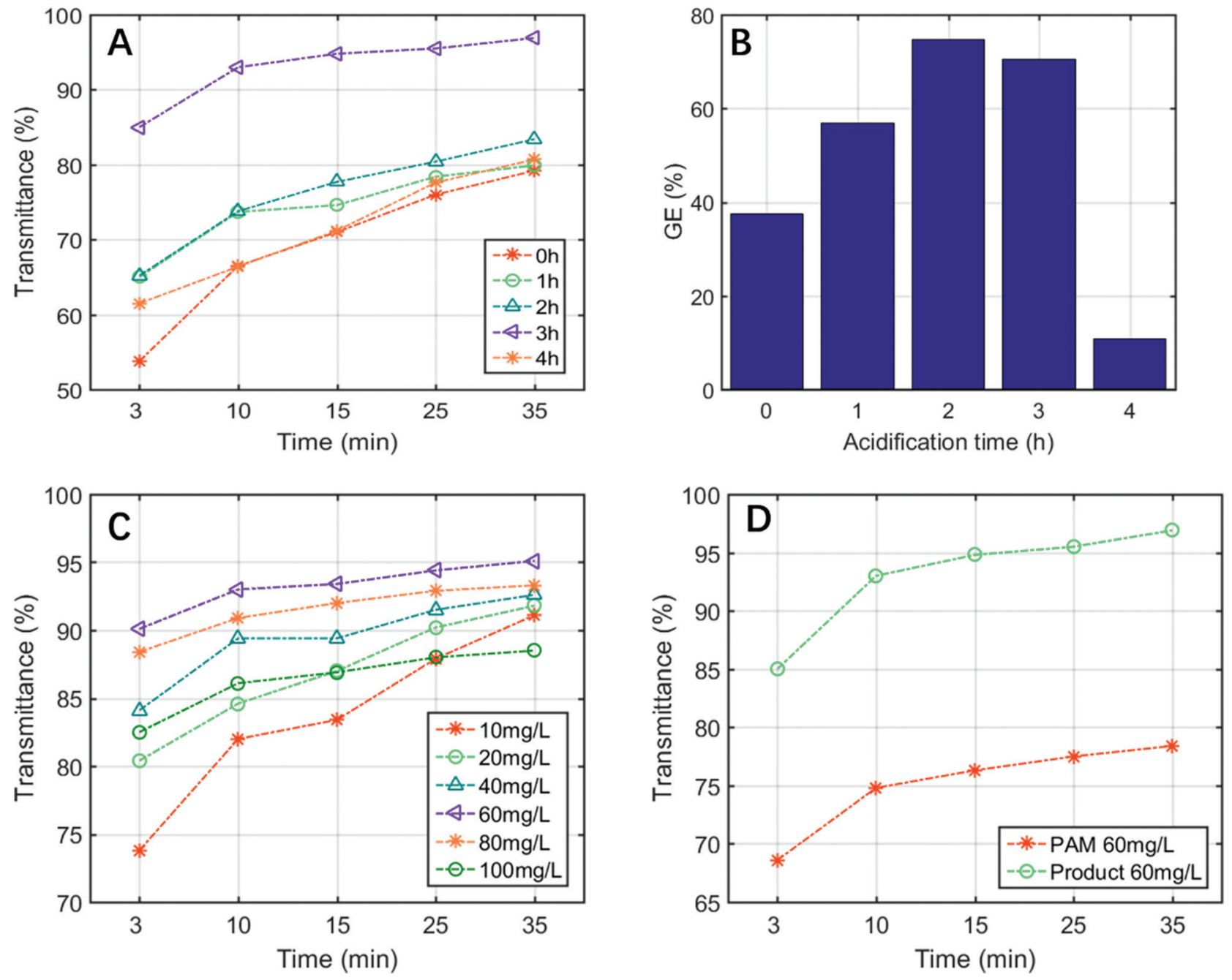

Figure 6: Effect of acidification time on transmittance (a) and GE (b); effect of flocculant dosage on transmittance (c); flocculation ability comparison (d).

CMC, which was confirmed by the characterizations. The results of EA, TG-DTG and SEM indicated that PAMD was actually grafted onto CMC, and FT-IR and NMR spectra demonstrated the successful formation of the polyamidine structure (five-membered cyclic copolymer). The optimal synthesis conditions of CMC-g-PAMD were experimentally determined: $4 \mathrm{~g}$ initiator dosage, $50^{\circ} \mathrm{C}$ polymerization temperature, $5 \mathrm{~h}$ polymerization time, $4 \mathrm{~h}$ acidification time, $90^{\circ} \mathrm{C}$ amidinization temperature and $3 \mathrm{~h}$ amidinization temperature. The new flocculant was employed in coalmine wastewater treatment, and the most suitable dosage was $60 \mathrm{mg} / \mathrm{L}$. Compared with the conventional flocculant (PAM), CMC-g-PAMD presented an overwhelming high turbidity removal performance with a transmittance of $96.1 \%$, which is bound to have broad prospects for wastewater treatment in the future.
Acknowledgments: This work was financially supported by the Local service fund of education department of Shaanxi province (18JF031), Xi’an science and technology innovation guidance project (201805041YD19CG25(4)), and Open Foundation of Shaanxi Key Laboratory of Lacustrine Shale Gas Accumulation and Exploitation (YJSYZX18SKF0003) was greatly acknowledged.

\section{References}

1. Yang Y.P., Yang Z.P., Xu G., Wang N.L., Situation and prospect of energy consumption for china's thermal power generation. Chin Soc Electr Eng, 2013, 33(23), 1-11.

2. Duan N., Guo J.P., Xie B.C., Is there a difference between the energy and $\mathrm{CO}_{2}$, emission performance for china's thermal power industry? A bootstrapped directional distance function approach. Appl Energy, 2016, 162, 1552-1563. 
3. National Bureau of Statistics of the People's Republic of China, China statistical yearbook China. Statistical Publishing House, Beijing, 2017.

4. Zeng Q., Li G.S., Dong J.X., Pu Y., Typical ecological and environmental issues and countermeasures in coal mining in Xinjiang Region. Min Saf Environ Prot, 2017, 44(1), 106-110.

5. He B., Li S.R., Effluent sewage recycling project in wangjiashan coal mine. Coal, 2018, 27(2), 0018-02.

6. Bian Z.F., Inyang H.I., Daniels J.L., Otto F., Environmental issues from coal mining and their solutions. Int J Min Sci Technol, 2010, 20(2), 215-223.

7. Chabukdhara M., Singh O.P., Coal mining in Northeast India: an overview of environmental issues and treatment approaches. Int J Coal Sci \& Technol, 2016, 3(2), 1-10.

8. Chai S.L., Robinson J., Mei F.C., A review on application of flocculants in wastewater treatment. Proc Saf Environ Prot, 2014, 92(6), 489-508.

9. Yan M.Q., Wang D.S., Yu J.F., Ni J.R., Edwards M., Qu J.H., Enhanced coagulation with polyaluminum chlorides: role of ph/alkalinity and speciation. Chemosphere, 2008, 71(9), 1665-1673.

10. Song L., Application of flocculants to water treatment and its forecast. Ind Water Treat, 2010, 30(6), 4-7.

11. Wang Q., Zhao L., Research progress of new inorganic polymer flocculants. Liaoning Chem Ind, 2017, 46(2), 0185-02.

12. Hou M.L., Wei Y.X., Wu Z., Liu H., Cao F.M., A study on the preparation and application of polymer ferric salt flocculants. Chem Propellants Polym Mater 2003, 1829(1), 151-157.

13. Hu F., Research progress of species of aluminum salt flocculants. Pap Sci Technol, 2007, 26(3), 0036-05.

14. Zhang P.Y., Wu Z., Zhang G.M., Zeng G.M., Zhang H., Li J., et al., Coagulation characteristics of polyaluminum chlorides pac-al 30, on humic acid removal from water. Sep Purif Technol, 2008, 63(3), 642-647.

15. Zhang M., Xiao F., Wang D.S., Xu X.Z., Zhou Q., Comparison of novel magnetic polyaluminum chlorides involved coagulation with traditional magnetic seeding coagulation: coagulant characteristics, treating effects, magnetic sedimentation efficiency and floc properties. Sep Purif Technol, 2017, 182, 118-127.

16. Kirwan L.J., Investigating bauxite residue flocculation by hydroxamate and polyacrylate flocculants utilising the focussed beam reflectance measurement probe. Int J Miner Process, 2009, 90(1), 74-80.

17. Gerchman Y., Vasker B., Tavasi M., Mishael Y., Kinel-Tahan Y., Yehoshua Y., Effective harvesting of microalgae: Comparison of different polymeric flocculants. Bioresour Technol, 2017, 228, 141-146.

18. Liu C., Hong B., Xu K., Zhang M.Y., An H.Y., Tan Y., et al., Synthesis and application of salt tolerance amphoteric hydrophobic associative flocculants. Polym Bull, 2014, 71(12), 3051-3065.

19. Zou Y.Q., Li S.S., Wang Y.Q., et al., Flocculation behavior of cationic pea starch prepared by the graft copolymerization of acrylamide for wastewater treatment. J Appl Polym Sci, 2016, 133(37), 43922-43911.

20. Li S.S., Zheng L., Wang Y.Q., Han X.L., Sun W., Yue Y.J., et al., Polyacrylamide-grafted legume starch for wastewater treatment: synthesis and performance comparison. Polym Bull, 2017, 74(11), 4371-4392.
21. Ochoa G.J.R., Muñoz H.M., Reinoso D., et al., Stability of inverse microemulsions of acrylamide-based anionic flocculants: evidence about the need of unsaturated surfactants. e-Polymers, 2008, 8(1), 320-329.

22. Ochoa G.J.R., Río F., Sasia P.M., et al., Synthesis of cationic flocculants based on acrylamide and (2-(acryloyloxy)ethyl) trimethylammonium chloride co polymers by semicontinuous inverse microemulsion co polymerization. Part I: Criteria for selection of comonomer formulation. e-Polymers, 2006, 6(1), 327-340.

23. Zhou H.J., Zhou L., Yang X.Y., Optimization of preparing a high yield and high cationic degree starch graft copolymer as environmentally friendly flocculant: Through response surface methodology. Int J Biol Macromol, 2018, 10(15), 1431-1437.

24. Huang M., Liu Z.Z., Li A.M., et al., Dual functionality of a graft starch flocculant: Flocculation and antibacterial performance. J Environ Manage, 2017, 196, 63-71.

25. Furusho Y., Endo T., Supramolecular polymer gels formed from carboxy-terminated telechelic polybutadiene and polyamidine through amidinium-carboxylate salt bridge. J Polym Sci, Part A: Polym Chem, 2014, 52(13), 1815-1824.

26. Furusho Y., Endo T., Capture and release of $\mathrm{CO}_{2}$, by polyamidine. J Polym Sci, Part A: Polym Chem, 2013, 51(16), 3404-3411.

27. Aleksandrova E.L., Dudkina M.M., Ten'Kovtsev A.V., Polyamidine supramolecular structures-a new class of photosensitive polymeric semiconductors. Semiconductors, 2003, 37(3), 266-270.

28. Guo B., Gao B.Y., Rong H.Y., et al., Coagulation performance and floc properties of compound bioflocculant-aluminum sulfate dual-coagulant in treating kaolin-humic acid solution. Chem Eng J, 2011, 173(2), 400-406.

29. Wang Y.Q., Yue Y.J., Ren J.H., et al., A polyamidine-based flocculant: synthesis and characterization. China Invention Patent: 201810434161.X.

30. Cai T., Yang Z., Yang H., Li A.M., Cheng R.S., Preparation and flocculation properties of carboxymethyl cellulose-graft-polyacrylamide. J Nanjing University, 2013, 49(4), 500-505.

31. Siqueira E.J., Salon M.B., Belgacem M.N., et al., Carboxymethylcellulose (CMC) as a model compound of cellulose fibers and polyamideamine epichlorohydrin (PAE)-CMC interactions as a model of PAE-fibers interactions of PAE-based wet strength papers. J Appl Polym Sci, 2015, 132(26), 42144 (1 of 10).

32. Sabaa M.W., Elzanaty A.M., Abdelgawad O.F., et al., Synthesis, characterization and antimicrobial activity of Schiff bases modified chitosan-graft-poly(acrylonitrile). Int J Biol Macromol, 2017, 109, 1280-1291.

33. Guo B., Yu H., Gao B.Y., et al., Novel cationic polyamidine: Synthesis, characterization, and sludge dewatering performance. J Environ Sci, 2017, 51(1), 305-314.

34. Zhao Y.X., Wang Z.T., The study on the synthesis of the starch grafted polyamidine. 2008.

35. Ghaemy M., Pour Z.S., Removal of dyes and heavy metal ions from water by magnetic hydrogel beads based on poly(vinyl alcohol)/carboxymethyl starch-g-poly(vinyl imidazole). Rsc Adv, 2015, 5(79), 64106-64118.

36. Reddy K.R., Hassan M., Gomes V.G., Hybrid nanostructures based on titanium dioxide for enhanced photocatalysis. Appl Catal A Gen, 2015, 489, 1-16. 